\title{
The Effect of Control Strength on Lag Synchronization of Nonlinear Coupled Complex Networks
}

\author{
Shuguo Wang ${ }^{1}$ and Hongxing Yao ${ }^{2,3}$ \\ ${ }^{1}$ Department of Mathematics and Physics, Changzhou Campus, Hohai University, \\ Jiangsu, Changzhou 213022, China \\ ${ }^{2}$ Faculty of Science, Jiangsu University, Jiangsu, Zhenjiang 212013, China \\ ${ }^{3}$ School of Finance and Economics, Jiangsu University, Jiangsu, Zhenjiang 212013, China \\ Correspondence should be addressed to Shuguo Wang,wsg97@163.com
}

Received 15 March 2012; Accepted 16 May 2012

Academic Editor: Tingwen Huang

Copyright (C) 2012 S. Wang and H. Yao. This is an open access article distributed under the Creative Commons Attribution License, which permits unrestricted use, distribution, and reproduction in any medium, provided the original work is properly cited.

This paper mainly investigates the lag synchronization of nonlinear coupled complex networks using methods that are based on pinning control, where the weight configuration matrix is not necessarily symmetric or irreducible. We change the control strength into a parameter concerning time $t$, by using the Lyapunov direct method, some sufficient conditions of lag synchronization are obtained. To validate the proposed method, numerical simulation examples are provided to verify the correctness and effectiveness of the proposed scheme.

\section{Introduction}

In recent years, a great deal of attention has been paid to the investigation of complex networks in various fields. In fact, complex networks are shown to widely exist in our life. Common examples of complex networks include the Internet, the World Wide Web (WWW), food webs, scientific citation webs, as well as many other systems that are made up of a large number of intricately connected parts. Indeed, complex networks are an important part of our daily lives.

Synchronization of complex networks has been one of the focal points in many research and application fields. Synchronization has been studied from various angles and a variety of different synchronization phenomena have been discovered, such as complete synchronization (CS), phase synchronization (PS), lag synchronization (LS), generalized 
synchronization (GS), anticipatory synchronization, antiphase synchronization, clustering synchronization, projective synchronization, and others [1-15]. It is worth mentioning that, in many practical situations, a propagation delay will appear in the electronic implementation of dynamical systems. Therefore, it is very important to investigate the lag synchronizationa few results have been reported. Guo [16] investigated the lag synchronization of complex networks via pinning control. Without assuming the symmetry and irreducibility of the coupling matrix, sufficient conditions of lag synchronization are obtained by adding controllers to a part of nodes. Particularly, the following two questions are solved: (1) How many controllers are needed to pin a coupled complex network to a homogeneous solution? (2) how should we distribute these controllers? Shahverdiev et al. [17] investigated lag synchronization between unidirectionally coupled Ikeda systems with time delay via feedback control techniques; Yang and Cao [18] studied the exponential lag synchronization of a class of chaotic delayed neural networks with impulsive effects. Some sufficient conditions are established by the stability analysis of impulsive differential equations. Li et al. [19] considered the lag synchronization issue of coupled time-delayed systems with chaos, applied proposed lag synchronization strategies towards the secure communication. Wang and Shi [20] investigated the chaotic bursting lag synchronization of Hindmarsh-Rose system via a single controller. Zhou et al. [21] investigated lag synchronization of coupled chaotic delayed neural networks without noise perturbation by using adaptive feedback control techniques. Wang et al. [22] investigated lag synchronization of chaotic systems with parameter mismatches. Sun and Cao [23] and Yu and Cao [24] researched the adaptive lag synchronization of unknown chaotic delayed neural networks.

It is noticed that almost all the regimes of lag synchronization mentioned above used the method of adding controllers to all the nodes to make complex networks get synchronized. As we know now, the real-world complex networks normally have a large number of nodes. Therefore, for the complexity of the dynamical network, it is difficult to realize the synchronization by adding controllers to all nodes. To reduce the number of the controllers, a natural way is using pinning control method [25-29].

Motivated by the above discussions, in this paper, we work on the lag synchronization of nonlinear coupled complex networks via pinning control method. The main contributions of this paper are three fold. (1) This paper deals with the lay synchronization problem for nonlinear coupled complex networks. We change the control strength into a parameter concerning time $t$, some sufficient conditions for the synchronization are derived by constructing an effective control scheme. Particularly, the weight configuration matrix is not necessarily symmetric or irreducible. (2) Compared with some similar designs, our pinning controllers are very simple. (3) Generally, previous works require the coupling strength $c$ to be large so that the synchronization of complex networks can be realized. However, there exists a drawback as $c$ becomes larger. This equivalently makes all weights larger simultaneously. This must raise the synchronization cost. In this paper, we show that, as a parameter, $\varepsilon(t)>0$ can be used to complete the task with a lower cost. Numerical examples are also provided to demonstrate the effectiveness of the theory. This work improves the current results that we have.

The rest of this paper is organized as follows. The network model is introduced and some necessary definitions, lemmas, and hypotheses are given in Section 2. The lag synchronization of the coupled complex networks is discussed in Section 3. Examples and their simulations are obtained in Section 4. Finally, conclusions are drawn in Section 5. 


\section{Model and Preliminaries}

Now we consider the nonlinear coupled complex networks consisting of $m$ identical nodes that are $n$-dimensional dynamical units. The model is described as

$$
\dot{x}_{i}(t)=f\left(t, x_{i}(t)\right)+c \sum_{j=1}^{m} a_{i j} g\left(x_{j}(t)\right), \quad i=1, \ldots, m,
$$

where $x_{i}(t)=\left(x_{i 1}(t), x_{i 2}(t), \ldots, x_{i n}(t)\right)^{T} \in R^{n}$ is the state vector of node $i ; f: R^{n} \rightarrow R^{n}$ standing for the activity of an individual subsystem is a vector value function. $g(\bullet)$ is some nonlinear function reflecting the nonlinear coupling relationship between those nodes. $A=\left(a_{i j}\right)_{m \times m}$ is the corresponding coupling matrix that satisfies $a_{i j} \geq 0(i \neq j)$, denoting the coupling coefficients, and $a_{i i}=-\sum_{j=1, j \neq i}^{m} a_{j i}$, for $i, j=1,2, \ldots, m$ and $c$ is the coupling strength and will be fixed in this paper.

Based on the system above, we construct a response system whose state variables are denoted by $y_{i}(i=1,2, \ldots, m)$, whereas (2.1) is considered as the drive system with state variables denoted by $x_{i}(i=1,2, \ldots, m)$. In the response network, we add controllers to a part of the nodes which will be much more practical. Without loss of generality, we add the controllers to the first $m_{1}$ nodes $\left(1 \leq m_{1} \leq m\right)$. Therefore, the response system with delay feedback can be described as

$$
\begin{gathered}
\dot{y}_{i}(t)=f\left(t, y_{i}(t)\right)+c \sum_{j=1}^{m} a_{i j} g\left(y_{j}(t)\right)-c \varepsilon(t)\left(g\left(y_{i}(t)\right)-g\left(x_{i}(t-\tau)\right)\right), \quad i=1, \ldots, m_{1} \\
\dot{y}_{i}(t)=f\left(t, y_{i}(t)\right)+c \sum_{j=1}^{m} a_{i j} g\left(y_{j}(t)\right), \quad i=m_{1}+1, \ldots, m,
\end{gathered}
$$

where $\tau>0$ is the time delay, $\varepsilon(t)>0$ and $\dot{\varepsilon}(t)=\sum_{i=1}^{m_{1}} \delta x_{i}^{T}(t) P \delta x_{i}(t)$. Define $\delta x_{i}(t)=y_{i}(t)-$ $x_{i}(t-\tau)$ and $\delta g\left(x_{i}(t)\right)=g\left(y_{i}(t)\right)-g\left(x_{i}(t-\tau)\right)$; then we have the error system as

$$
\delta \dot{x}_{i}(t)=f\left(t, y_{i}(t)\right)-f\left(t, x_{i}(t-\tau)\right)+c \sum_{j=1}^{m} \tilde{a}_{i j} \delta g\left(x_{j}(t)\right), \quad i=1, \ldots, m
$$

where $\tilde{a}_{i i}=a_{i i}-\varepsilon(t), i=1, \ldots, m_{1}$ and $\tilde{a}_{i j}=a_{i j}$ otherwise.

Now, we introduce some definitions, assumptions, and lemmas that will be required throughout the paper.

Definition 2.1 (see [30]). The drive system (2.1) is said to lag synchronize with the response system (2.2) at time $\tau$ if $y_{i}(t)-x_{i}(t-\tau) \rightarrow 0, t \rightarrow \infty, i=1, \ldots, m$, where $\tau$ is a given positive time delay.

Lemma 2.2 (see [31]). Assuming that $A=\left(a_{i j}\right)_{n \times n}$ satisfies the following conditions.

(1) $a_{i j} \geq 0,(i \neq j), a_{i i}=-\sum_{j=1, i \neq j}^{n} a_{i j}, i, j=1,2, \ldots, n$. 
(2) $A$ is irreducible. Then, one has

(i) real parts of the eigenvalues of $A$ are all negative except an eigenvalue 0 with multiplicity 1 ,

(ii) $A$ has the right eigenvector $(1,1, \ldots, 1)^{T}$ corresponding to the eigenvalue 0 ,

(iii) let $\xi=\left(\xi_{1}, \xi_{2}, \ldots, \xi_{n}\right)^{T}$ be the left eigenvector of $A$ corresponding to the eigenvalue $0, \xi_{i}>0, i=1, \ldots n$ for convenience, one writes $\Xi=\operatorname{diag}\left\{\xi_{1}, \ldots, \xi_{n}\right\}$.

Lemma 2.3 (see [32]). If $A=\left(a_{i j}\right)_{n \times n}$ is an irreducible matrix and satisfies $a_{i j}=a_{j i} \geq 0$, for $i \neq j$, and $a_{i i}=-\sum_{j=1, i \neq j}^{n} a_{i j}, i, j=1,2, \ldots, n$ then, all eigenvalues of the matrix $\tilde{A}=A-$ $\operatorname{diag}\left(k_{1}, k_{2}, \ldots, k_{m_{1}}, 0, \ldots, 0\right)$ are negative, where $k_{1}, k_{2}, \ldots, k_{m_{1}}$ are positive constants.

Assumption 2.4 (see [33]). The function $f(\bullet) \in Q U A D(P, \Delta, \eta)$ if there exists a positive definite diagonal matrix $P=\operatorname{diag}\left(p_{1}, \ldots, p_{n}\right)$, a diagonal matrix $\Delta=\operatorname{diag}\left(\Delta_{1}, \ldots, \Delta_{n}\right)$, and a scalar $\eta>0$ such that $(x-y)^{T} P(f(x)-f(y)-\Delta x+\Delta y) \leq-\eta(x-y)^{T}(x-y)$ holds for any $x, y \in R_{n}, t>0$.

Assumption 2.5 (see [34] (Global Lipschitz Condition)). Suppose that there exist nonnegative constants $\gamma$, for all $\forall t \in R_{+}$, such that for any time-varying vectors $x(t), y(t) \in R^{n}$

$$
\|g(x)-g(y)\| \leq \gamma\|x-y\|
$$

where $\|||$ denotes the 2-norm throughout the paper.

For the convenience of later use, we introduce some notations:

$$
\begin{gathered}
\delta x(t)=\left[\delta x_{1}(t)^{T}, \ldots, \delta x_{m}(t)^{T}\right]^{T}, \quad \delta \tilde{x}^{k}(t)=\left[\delta x_{1}^{k}(t), \ldots, \delta x_{m}^{k}(t)\right]^{T}, \quad k=1, \ldots, n, \\
\delta g\left(\tilde{x}^{k}(t)\right)=\left[\delta g\left(x_{1}^{k}(t)\right), \ldots, \delta g\left(x_{m}^{k}(t)\right)\right], \quad k=1, \ldots, n .
\end{gathered}
$$

\section{Main Results}

According to proposition in [16], we can get that the matrix $\Xi A$ is zero row sum. Moreover, due to $A$ being an irreducible coupling matrix and $\Xi$ a positive diagonal matrix, it is easy to verify that $\Xi A$ is also irreducible and the matrix $\Xi A$ is negative definite.

Theorem 3.1. Suppose that Assumptions 2.4 and 2.5 hold and the coupling matrix $A$ is irreducible. If one has

$$
\Delta_{k} \Xi+c \gamma(\Xi \tilde{A}) \leq 0, \quad k=1, \ldots, n
$$

then, the drive system (2.1) lag synchronization with the response system (2.2) at time $\tau$.

Proof. Choose the following Lyapunov functional candidate:

$$
V(t)=\frac{1}{2} \sum_{i=1}^{m} \xi_{i} \delta x_{i}^{T}(t) P \delta x_{i}(t)
$$


Differentiating $V(t)$ with respect to time along the solution of (2.3) yields

$$
\begin{aligned}
\dot{V}(t) & =\sum_{i=1}^{m} \xi_{i} \delta x_{i}^{T}(t) P \delta \dot{x}_{i}^{T}(t) \\
& =\sum_{i=1}^{m} \xi_{i} \delta x_{i}^{T}(t) P\left[f\left(t, y_{i}(t)\right)-f\left(t, x_{i}(t-\tau)\right)+c \sum_{j=1}^{m} \tilde{a}_{i j} \delta g\left(x_{j}(t)\right)\right] .
\end{aligned}
$$

By the Assumption 2.4 and Lemmas 2.2 and 2.3, we obtain

$$
\begin{aligned}
\dot{V}(t) & \leq-\eta \sum_{i=1}^{m} \xi_{i} \delta x_{i}^{T}(t) \delta x_{i}(t)+\sum_{i=1}^{m} \xi_{i} \delta x_{i}^{T}(t) P \Delta \delta x_{i}(t)+c \sum_{i=1}^{m} \xi_{i} \delta x_{i}^{T}(t) P \sum_{j=1}^{m} \tilde{a}_{i j} \delta g\left(x_{j}(t)\right) \\
& \leq-\eta \sum_{i=1}^{m} \xi_{i} \delta x_{i}^{T}(t) \delta x_{i}(t)+\sum_{k=1}^{n} p_{k} \Delta_{k}\left(\delta \tilde{x}^{k}(t)\right)^{T} \Xi \delta \tilde{x}^{k}(t)+c \sum_{k=1}^{n} p_{k}\left(\delta \tilde{x}^{k}(t)\right)^{T} \Xi \tilde{A} \delta g\left(\tilde{x}^{k}(t)\right) .
\end{aligned}
$$

By the Assumption 2.5, we obtain

$$
\dot{V}(t) \leq-\eta \sum_{i=1}^{m} \xi_{i} \delta x_{i}^{T}(t) \delta x_{i}(t)+\sum_{k=1}^{n} p_{k}\left(\delta \tilde{x}^{k}(t)\right)^{T}\left[\Delta_{k} \Xi+c r(\Xi \tilde{A})\right] \delta \tilde{x}^{k}(t) .
$$

Therefore, if we have $\Delta_{k} \Xi+c \gamma(\Xi \tilde{A}) \leq 0, k=1, \ldots, n$ then

$$
\dot{V}(t) \leq 0
$$

Theorem 3.1 is proved completely.

Theorem 3.2. Suppose that Assumptions 2.4 and 2.5 hold and the coupling matrix $A$ is reducible. If one has when

$$
\Delta_{k} \Xi+c \gamma \Xi A-c q \Lambda<0 \quad k=1, \ldots, n
$$

where $\Lambda=\left(\begin{array}{cc}I_{m_{1} \times m_{1}} & 0 \\ 0 & 0\end{array}\right)_{m \times m}$, then, the drive system (2.1) lag synchronize with the response system (2.2) at time $\tau$.

Proof. We consider the following system:

$$
\begin{gathered}
\delta x_{i}(t)=f\left(t, y_{i}(t)\right)-f\left(t, x_{i}(t-\tau)\right)+c \sum_{j=1}^{m} a_{i j} \delta g\left(x_{j}(t)\right) \\
-c \varepsilon(t)\left(g\left(y_{i}(t)\right)-g\left(x_{i}(t-\tau)\right)\right), \quad i=1, \ldots, m_{1} \\
\delta x_{i}(t)=f\left(t, y_{i}(t)\right)-f\left(t, x_{i}(t-\tau)\right)+c \sum_{j=1}^{m} a_{i j} \delta g\left(x_{j}(t)\right), \quad i=m_{1}+1, \ldots, m .
\end{gathered}
$$


Choose the following Lyapunov functional candidate:

$$
V(t)=\frac{1}{2} \sum_{i=1}^{m} \xi_{i} \delta x_{i}^{T}(t) P \delta x_{i}(t)+\frac{c}{2} \sum_{i=1}^{m_{1}} \frac{\left(\gamma \xi_{i} \varepsilon(t)-q\right)^{2}}{\gamma \xi_{i}}
$$

where $q>0$.

Differentiating $V_{1}(t)$ with respect to time along the solution of (3.8) yields

$$
\begin{aligned}
& \dot{V}(t)= \sum_{i=1}^{m} \xi_{i} \delta x_{i}^{T}(t) P \delta \dot{x}_{i}^{T}(t)+c \sum_{i=1}^{m_{1}}\left(\gamma \xi_{i} \varepsilon(t)-q\right) \dot{\varepsilon}(t) \\
&= \sum_{i=1}^{m} \xi_{i} \delta x_{i}^{T}(t) P\left[f\left(t, y_{i}(t)\right)-f\left(t, x_{i}(t-\tau)\right)+c \sum_{i=1}^{m} a_{i j} \delta g\left(x_{j}(t)\right)\right. \\
&\left.-c \varepsilon(t)\left(g\left(y_{i}(t)\right)-g\left(x_{i}(t-\tau)\right)\right)\right]+c \sum_{i=1}^{m_{1}}\left(\gamma_{i} \varepsilon(t)-q\right) \dot{\varepsilon}(t) \\
&=\sum_{i=1}^{m} \xi_{i} \delta x_{i}^{T}(t) P\left[f\left(t, y_{i}(t)\right)-f\left(t, x_{i}(t-\tau)\right)-\Delta \delta x_{i}(t)\right]+\sum_{i=1}^{m} \xi_{i} \delta x_{i}^{T}(t) P \Delta \delta x_{i}(t) \\
&+c \sum_{i=1}^{m} \xi_{i} \delta x_{i}^{T}(t) P \sum_{i=1}^{m} a_{i j} \delta g\left(x_{j}(t)\right)+c \sum_{i=1}^{m_{1}}\left(\gamma \xi_{i} \varepsilon(t)-q\right) \dot{\varepsilon}(t) \\
& \quad-c \sum_{i=1}^{m_{1}} \xi_{i} \delta x_{i}^{T}(t) P \varepsilon(t)\left(g\left(y_{i}(t)\right)-g\left(x_{i}(t-\tau)\right)\right)
\end{aligned}
$$

By the Assumption 2.4, we obtain

$$
\begin{aligned}
\dot{V}(t) \leq- & \eta \sum_{i=1}^{m} \xi_{i} \delta x_{i}^{T}(t) \delta x_{i}(t)+\sum_{k=1}^{n} p_{k}\left(\delta \tilde{x}^{k}(t)\right)^{T}\left(\Delta_{k} \Xi\right) \delta \tilde{x}^{k}(t)+c \sum_{i=1}^{m} \xi_{i} \delta x_{i}^{T}(t) P \sum_{i=1}^{m} a_{i j} \delta g\left(x_{j}(t)\right) \\
& -c \gamma \sum_{i=1}^{m_{1}} \xi_{i} \delta x_{i}^{T}(t) P \varepsilon(t) \delta x_{i}(t)+c \gamma \sum_{i=1}^{m_{1}} \xi_{i} \varepsilon(t) \dot{\varepsilon}(t)-c \sum_{i=1}^{m_{1}} q \dot{\varepsilon}(t) \\
\leq- & \eta \sum_{i=1}^{m} \xi_{i} \delta x_{i}^{T}(t) \delta x_{i}(t)+\sum_{k=1}^{n} p_{k}\left(\delta \tilde{x}^{k}(t)\right)^{T}\left(\Delta_{k} \Xi\right) \delta \tilde{x}^{k}(t) \\
& +c \sum_{k=1}^{n} p_{k}\left(\delta \tilde{x}^{k}(t)\right)^{T}(\Xi A) \delta g\left(\tilde{x}^{k}(t)\right)-c q \sum_{k=1}^{n} p_{k}\left(\delta \tilde{x}^{k}(t)\right)^{T} \Lambda\left(\delta \tilde{x}^{k}(t)\right) .
\end{aligned}
$$


By the Assumption 2.5, we obtain

$$
\dot{V}(t) \leq-\eta \sum_{i=1}^{m} \xi_{i} \delta x_{i}^{T}(t) \delta x_{i}(t)+\sum_{k=1}^{n} p_{k}\left(\delta \tilde{x}^{k}(t)\right)^{T}\left(\Delta_{k} \Xi+c \gamma \Xi A-c q \Lambda\right)\left(\delta \tilde{x}^{k}(t)\right) .
$$

Therefore, if we have $\Delta_{k} \Xi+c \gamma \Xi A-c q \Lambda<0, k=1, \ldots, n$ then

$$
\dot{V}(t) \leq 0,
$$

Theorem 3.2 is proved completely.

Remark 3.3. Compared with the control methods in the literature [16], the work requires the coupling strength $c$ and $k_{i}\left(u_{i}(t)=k_{i}\left(x_{i}(t-\tau)-y_{i}(t)\right)\right.$, where $k_{i}$ are positive constants) to be large so that the lag synchronization of complex networks can be realized. However, there exists a drawback as $c$ becomes larger. This equivalently makes all weights larger simultaneously. This must raise the synchronization cost. In this paper, we show that, as a parameter, $\varepsilon(t)>0$ can be used to complete the task with a lower cost.

\section{Illustrative Examples}

In this section, a numerical example will be given to demonstrate the validity of the lag synchronization criteria obtained in the previous sections. Considering the following network:

$$
\begin{gathered}
\dot{y}_{i}(t)=f\left(t, y_{i}(t)\right)+c \sum_{j=1}^{m} a_{i j} g\left(y_{j}(t)\right)-c \varepsilon(t)\left(g\left(y_{i}(t)\right)-g\left(x_{i}(t-\tau)\right)\right), \quad i=1, \ldots, m_{1} \\
\dot{y}_{i}(t)=f\left(t, y_{i}(t)\right)+c \sum_{j=1}^{m} a_{i j} g\left(y_{j}(t)\right), \quad i=m_{1}+1, \ldots, m,
\end{gathered}
$$

where $i=1,2, \ldots, m, f\left(t, y_{i}(t)\right)=D y_{i}(t)+h\left(y_{i}(t)\right)+B, y_{i}(t)=\left(y_{i 1}(t), y_{i 2}(t), y_{i 3}(t)\right)^{T}$, Here $B=[0,0,0.1]^{T}, h\left(x_{i}\right)=\left(0,0, y_{i 1} y_{i 3}\right)^{T}, m_{1}=1, c=0.5, \tau=0.01, g(y)=\cos y+3 y$. And

$$
D=\left[\begin{array}{ccc}
0 & -1 & -1 \\
1 & 0.1 & 0 \\
1 & 0 & -10
\end{array}\right], \quad A=\left[\begin{array}{cccccc}
-6 & 1 & 2 & 1 & 1 & 1 \\
1 & -5 & 2 & 1 & 0 & 1 \\
2 & 2 & -7 & 0 & 1 & 2 \\
1 & 1 & 0 & -7 & 2 & 3 \\
1 & 0 & 1 & 2 & -5 & 1 \\
1 & 1 & 2 & 3 & 1 & -8
\end{array}\right]
$$




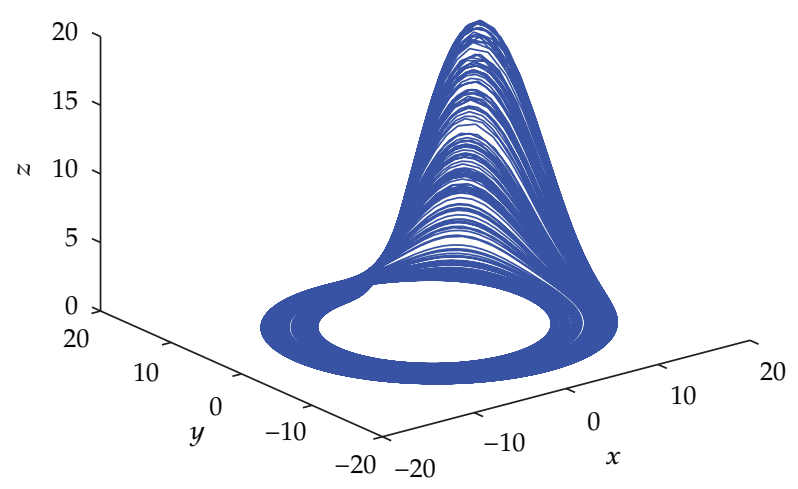

Figure 1: The chaotic behavior of time-delayed Rossler system.

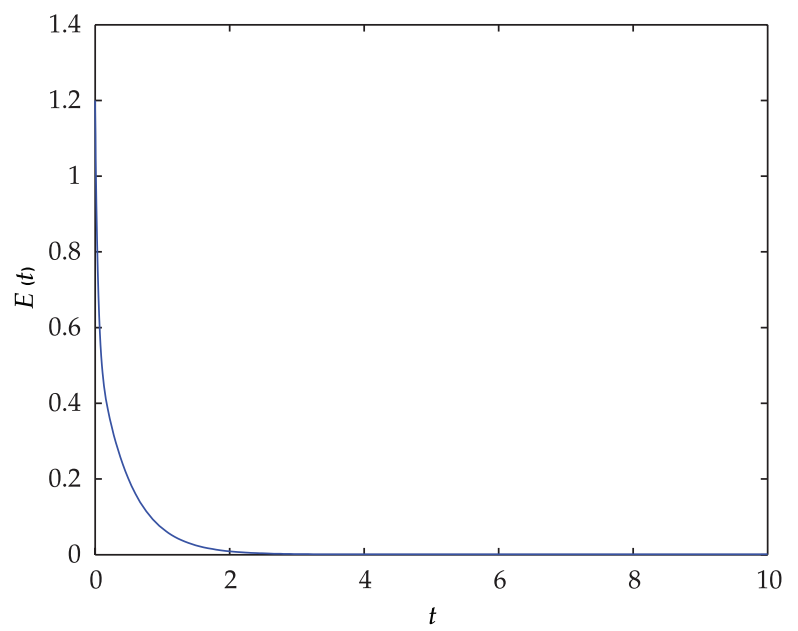

Figure 2: Time evolution of the lag synchronization errors $E(t)$.

The following quantities are utilized to measure the process of lag synchronization

$$
\begin{aligned}
& E(t)=\sum_{i=1}^{N}\left\|y_{i}(t)-x_{i}(t-\tau)\right\| \\
& e_{1}(t)=\left\|y_{1}(t)-x_{1}(t-\tau)\right\|,
\end{aligned}
$$

where $E(t)$ is the error of lag synchronization for this controlled network (2.2); $e_{1}(t)$ is used to display the synchronization process of the first pinned node. The simulation results are given in Figures 1, 2, 3, and 4. From Figure 4, we see the time evolution of control strength. The numerical results show that the theoretical results are effective.

Remark 4.1. In this paper we designed controllers to ensure that the special networks could get lag synchronization. It indeed provides some new insights for the future practical engineering design. 


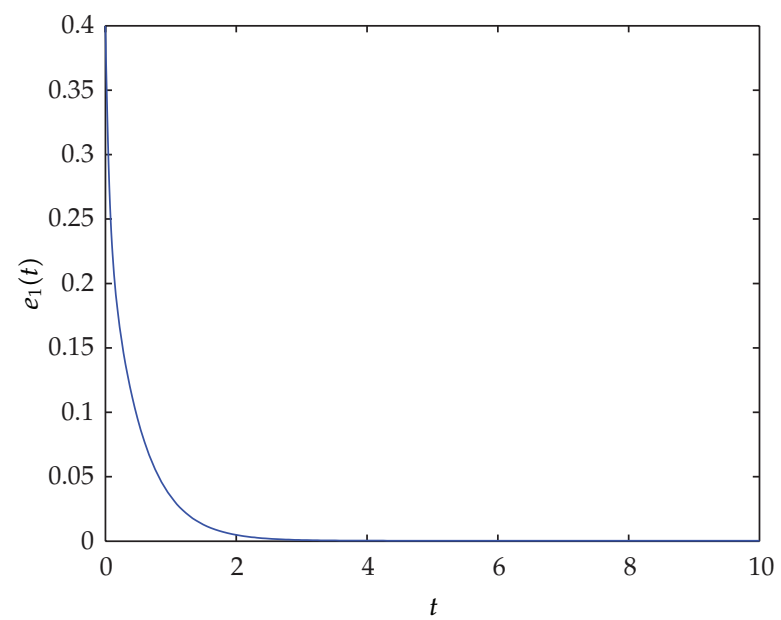

Figure 3: Time evolution of the lag synchronization errors $e_{1}(t)$.

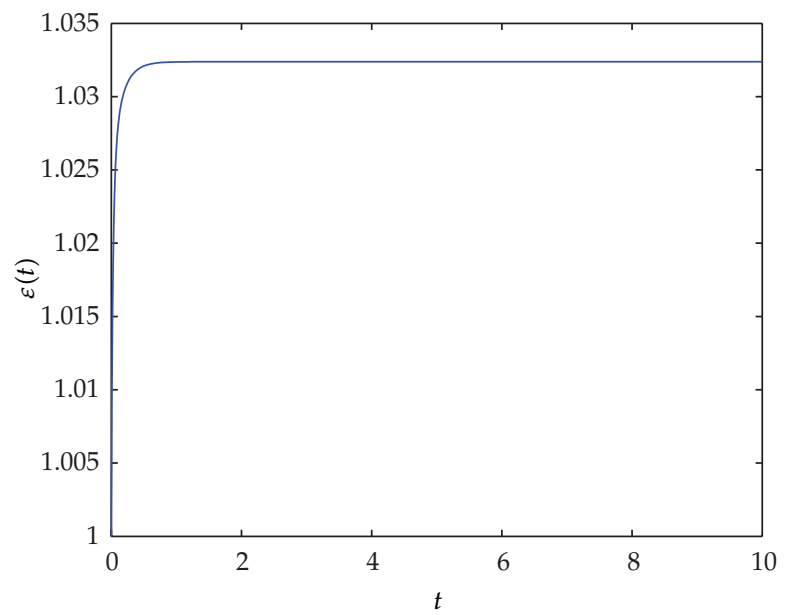

Figure 4: Time evolution of control strength $\varepsilon(t)$.

\section{Conclusions}

The problems of lag synchronization and pinning control for the nonlinear coupled complex networks are investigated. It is shown that lag synchronization can be realized via pinning controller. The study showed that the use of simple control law helps to derive sufficient criteria which ensure that the lag synchronization of the network model is derived. In addition, numerical simulations were performed to verify the effectiveness of the theoretical results.

\section{Acknowledgments}

Research is partially supported by the National Nature Science Foundation of China (no. 70871056) and by the Six Talents Peak Foundation of Jiangsu Province. 


\section{References}

[1] G. M. Mahmoud and E. E. Mahmoud, "Complete synchronization of chaotic complex nonlinear systems with uncertain parameters," Nonlinear Dynamics, vol. 62, no. 4, pp. 875-882, 2010.

[2] S. Xu and Y. Yang, "Global asymptotical stability and generalized synchronization of phase synchronous dynamical networks," Nonlinear Dynamics, vol. 59, no. 3, pp. 485-496, 2010.

[3] J. L. Wang and H. N. Wu, "Local and global exponential output synchronization of complex delayed dynamical networks," Nonlinear Dynamics, vol. 67, no. 1, pp. 497-504, 2012.

[4] Y. C. Hung, Y. T. Huang, M. C. Ho, and C. K. Hu, "Paths to globally generalized synchronization in scale-free networks," Physical Review E, vol. 77, no. 1, Article ID 016202, 8 pages, 2008.

[5] H. N. Agiza, "Chaos synchronization of Lü dynamical system," Nonlinear Analysis, vol. 58, no. 1-2, pp. 11-20, 2004.

[6] N. F. Rulkov, M. M. Sushchik, L. S. Tsimring, and H. D. I. Abarbanel, "Generalized synchronization of chaos in directionally coupled chaotic systems," Physical Review E, vol. 51, no. 2, pp. 980-994, 1995.

[7] M. G. Rosenblum, A. S. Pikovsky, and J. Kurths, "Phase synchronization of chaotic oscillators," Physical Review Letters, vol. 76, no. 11, pp. 1804-1807, 1996.

[8] L. P. Zhang, H. B. Jiang, and Q. S. Bi, "Reliable impulsive lag synchronization for a class of nonlinear discrete chaotic systems," Nonlinear Dynamics, vol. 59, no. 4, pp. 529-534, 2010.

[9] Z.-L. Wang, "Projective synchronization of hyperchaotic Lü system and Liu system," Nonlinear Dynamics, vol. 59, no. 3, pp. 455-462, 2010.

[10] Q. J. Zhang and J. C. Zhao, "Projective and lag synchronization between general complex networks via impulsive control," Nonlinear Dynamics, vol. 67, no. 4, pp. 2519-2525, 2012.

[11] Q. Han, C. D. Li, and J. J. Huang, "Anticipating synchronization of chaotic systems with time delay and parameter mismatch," Chaos, vol. 19, no. 1, Article ID 013104, 2009.

[12] L. Y. Cao and Y. C. Lai, "Anti-phase synchronization in chaotic systems," Physical Review E, vol. 58, pp. C382-C386, 1998.

[13] J. Y. Wang, J. W. Feng, C. Xu, and Y. Zhao, “Cluster synchronization of nonlinearly-coupled complex networks with nonidentical nodes and asymmetrical coupling matrix," Nonlinear Dynamics, vol. 67, no. 2, pp. 1635-1646, 2012.

[14] I. Belykh, V. Belykh, K. Nevidin, and M. Hasler, "Persistent clusters in lattices of coupled nonidentical chaotic systems," Chaos, vol. 13, no. 1, pp. 165-178, 2003.

[15] C. F. Feng, "Projective synchronization between two different time-delayed chaotic systems using active control approach," Nonlinear Dynamics, vol. 62, no. 1-2, pp. 453-459, 2010.

[16] W. L. Guo, "Lag synchronization of complex networks via pinning control," Nonlinear Analysis, vol. 12, no. 5, pp. 2579-2585, 2011, http://www.elsevier.com/locate/nonrwa.

[17] E. M. Shahverdiev, S. Sivaprakasam, and K. A. Shore, "Lag synchronization in time-delayed systems," Physics Letters A, vol. 292, no. 6, pp. 320-324, 2002.

[18] Y. Yang and J. Cao, "Exponential lag synchronization of a class of chaotic delayed neural networks with impulsive effects," Physica A, vol. 386, no. 1, pp. 492-502, 2007.

[19] C. Li, X. Liao, and K. Wong, "Chaotic lag synchronization of coupled time-delayed systems and its applications in secure communication," Physica D, vol. 194, no. 3-4, pp. 187-202, 2004.

[20] Z.-L. Wang and X.-R. Shi, "Chaotic bursting lag synchronization of Hindmarsh-Rose system via a single controller," Applied Mathematics and Computation, vol. 215, no. 3, pp. 1091-1097, 2009.

[21] J. Zhou, T. Chen, and L. Xiang, "Chaotic lag synchronization of coupled delayed neural networks and its applications in secure communication," Circuits, Systems, and Signal Processing, vol. 24, no. 5, pp. 599-613, 2005.

[22] L. P. Wang, Z. T. Yuan, X. H. Chen, and Z. F. Zhou, “Lag synchronization of chaotic systems with parameter mismatches," Communications in Nonlinear Science and Numerical Simulation, vol. 16, no. 2, pp. 987-992, 2011.

[23] Y. Sun and J. Cao, "Adaptive lag synchronization of unknown chaotic delayed neural networks with noise perturbation," Physics Letters A, vol. 364, no. 3-4, pp. 277-285, 2007.

[24] W. Yu and J. Cao, "Adaptive synchronization and lag synchronization of uncertain dynamical system with time delay based on parameter identification," Physica A, vol. 375, no. 2, pp. 467-482, 2007.

[25] W. L. Guo, F. Austin, S. H. Chen, and W. Sun, "Pinning synchronization of the complex networks with non-delayed and delayed coupling," Physics Letters A, vol. 373, no. 17, pp. 1565-1572, 2009. 
[26] X. F. Wang and G. Chen, "Pinning control of scale-free dynamical networks," Physica A, vol. 310, no. 3-4, pp. 521-531, 2002.

[27] T. Chen, X. Liu, and W. Lu, “Pinning complex networks by a single controller," IEEE Transactions on Circuits and Systems. I, vol. 54, no. 6, pp. 1317-1326, 2007.

[28] W. W. Yu, G. R. Chen, and J. H. Lü, "On pinning synchronization of complex dynamical networks," Automatica A, vol. 45, no. 2, pp. 429-435, 2009.

[29] X. Zhou, H. Feng, and S. Chen, "The effect of control strength on the synchronization in pinning control questions," Computers E Mathematics with Applications, vol. 61, no. 8, pp. 2014-2018, 2011.

[30] W. Yu and J. Cao, "Adaptive synchronization and lag synchronization of uncertain dynamical system with time delay based on parameter identification," Physica A, vol. 375, no. 2, pp. 467-482, 2007.

[31] W. Lu and T. Chen, "New approach to synchronization analysis of linearly coupled ordinary differential systems," Physica D, vol. 213, no. 2, pp. 214-230, 2006.

[32] W. Guo, F. Austin, S. Chen, and W. Sun, "Pinning synchronization of the complex networks with non-delayed and delayed coupling," Physics Letters A, vol. 373, no. 17, pp. 1565-1572, 2009.

[33] C. W. Wu, "Synchronization in arrays of coupled nonlinear systems with delay and nonreciprocal time-varying coupling," IEEE Transactions on Circuits and Systems II, vol. 52, no. 5, pp. 282-286, 2005.

[34] H. Liu, J. Chen, J. A. Lu, and M. Cao, "Generalized synchronization in complex dynamical networks via adaptive couplings," Physica A, vol. 389, no. 8, pp. 1759-1770, 2010. 


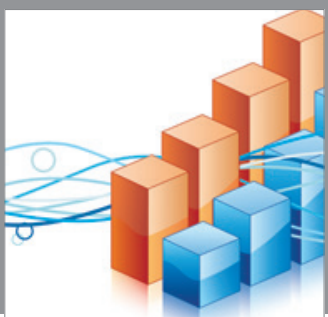

Advances in

Operations Research

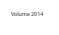

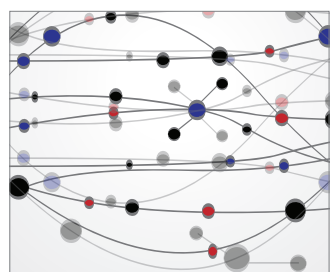

\section{The Scientific} World Journal
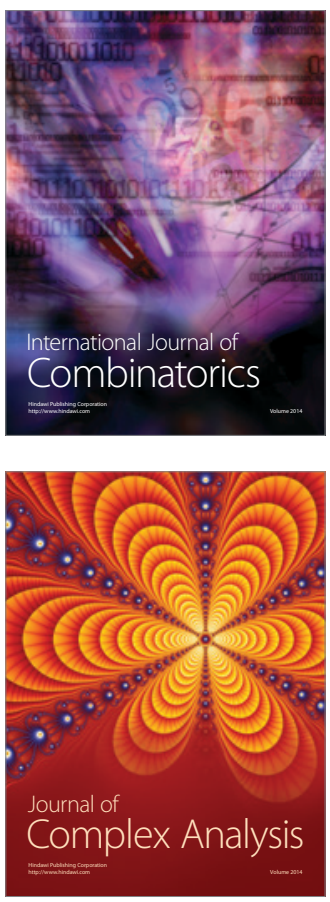

International Journal of

Mathematics and

Mathematical

Sciences
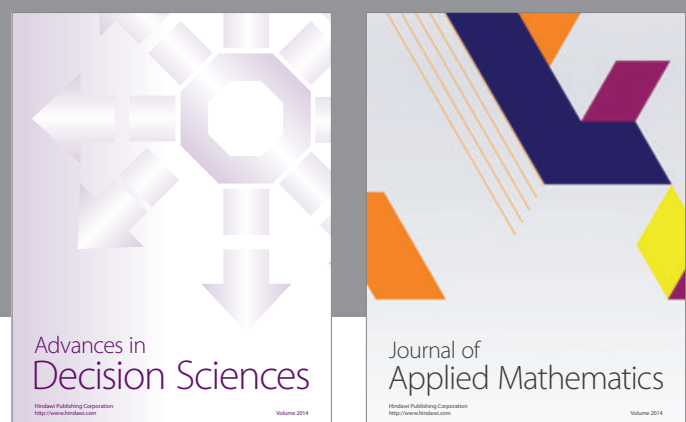

Journal of

Applied Mathematics
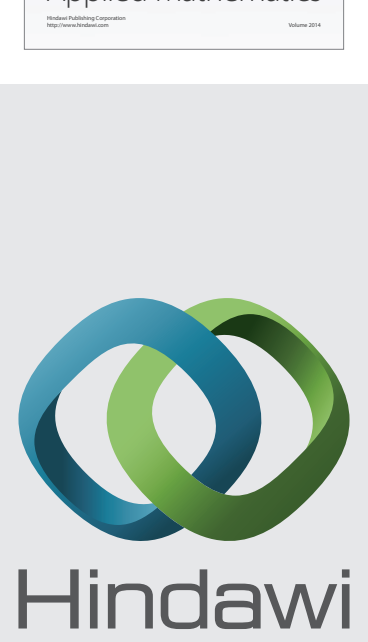

Submit your manuscripts at http://www.hindawi.com
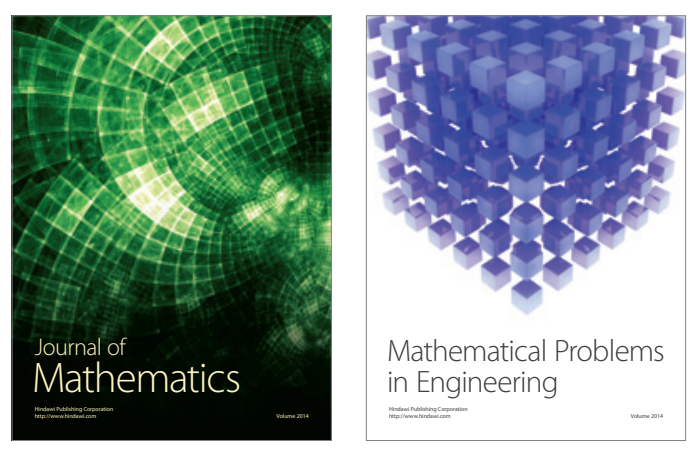

Mathematical Problems in Engineering
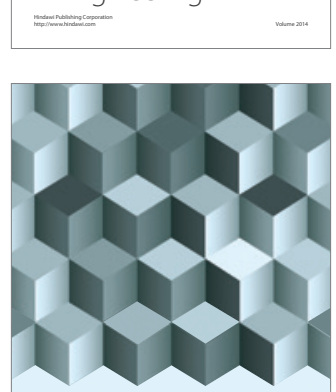

Journal of

Function Spaces
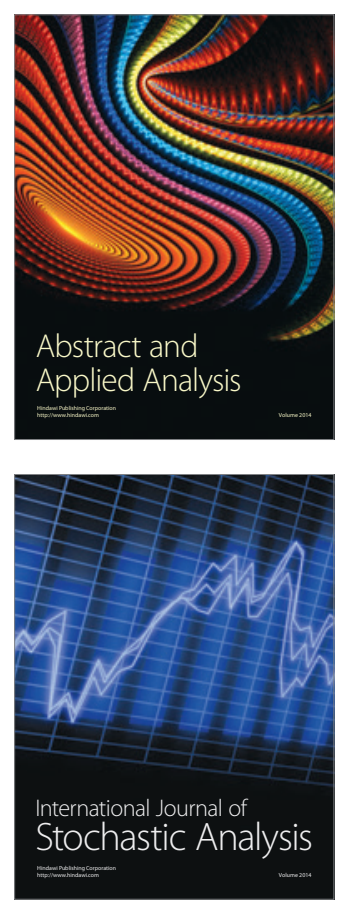

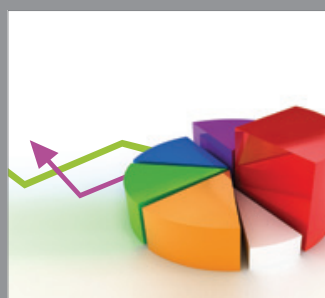

ournal of

Probability and Statistics

Promensencen
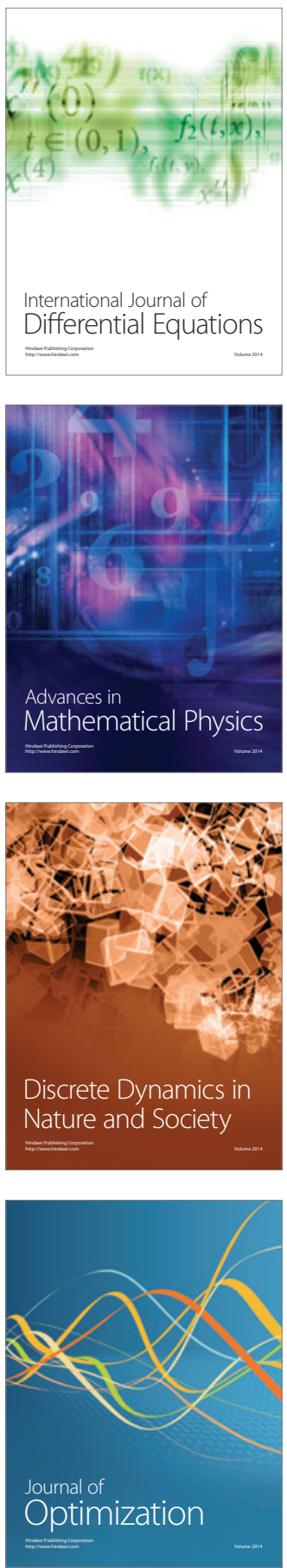